NBER WORKING PAPER SERIES

\title{
ELEMENTS OF A THEORY OF \\ DESIGN LIMITS TO OPTIMAL POLICY
}

\author{
William A. Brock \\ Steven N. Durlauf \\ Working Paper 10495 \\ http://www.nber.org/papers/w10495
}

\section{NATIONAL BUREAU OF ECONOMIC RESEARCH 1050 Massachusetts Avenue Cambridge, MA 02138}

May 2004

\begin{abstract}
An early version of this paper was delivered by Brock as the 2003 Harry Johnson Memorial Lecture at Cambridge University. The authors thank the National Science Foundation, John D. and Catherine T. MacArthur Foundation, Vilas Trust and University of Wisconsin for financial support and Ritesh Banerjee, Ethan Cohen-Cole and Giacomo Rondina for excellent research assistance. Thanks to Soren Johansen and audience members at Cambridge University, Columbia University, London Business School, the Federal Reserve Bank of Atlanta, the Federal Reserve Board of Governors and the Middlebury Conference on PostWalrasian Economics for comments on a previous draft. The views expressed herein are those of the author(s) and not necessarily those of the National Bureau of Economic Research.
\end{abstract}

(C2004 by William A. Brock and Steven N. Durlauf. All rights reserved. Short sections of text, not to exceed two paragraphs, may be quoted without explicit permission provided that full credit, including $(\mathrm{C}$ notice, is given to the source. 
Elements of a Theory of Design Limits to Optimal Policy

William A. Brock and Steven N. Durlauf

NBER Working Paper No. 10495

May 2004

JEL No. C52, E6

\section{$\underline{\text { ABSTRACT }}$}

This paper presents a framework for understanding the limits that exist in optimal policy design in dynamic contexts. We consider the design of policies in the context of dynamic linear models. Fundamental design limits exist for policy rules in such environments in the sense that any policy rule embodies tradeoffs between the magnitudes of different frequency-specific components of the variance. Hence policies that are effective in eliminating low frequency variance components of a state variable can only do so at the cost of exacerbating high frequency variance components, and vice versa. Examples of the implications of such tradeoffs are considered.

William A. Brock

Department of Economics

University of Wisconsin

1180 Observatory Drive

Madison, WI 53706-1393

wbrock@ssc.wisc.edu

Steven N. Durlauf

Department of Economics

University of Wisconsin

1180 Observatory Drive

Madison, WI 53706-1393

and NBER

sdurlauf@ssc.wisc.edu 
"The role of the economists in discussions of public policy seems to me to be to prescribe what should be done in light of what can be done, politics aside..."

Milton Friedman (1951,pg. 187)

\section{Introduction}

This paper studies a number of issues related to the design of optimal policies in dynamic contexts. Issues of policy design have experienced a renaissance in macroeconomics over the last decade, driven to a significant extent by the modern literature on monetary policy rules, a literature that was to an important extent initiated by Taylor (1993); Taylor (1999) contains a wide range of applications. An important feature of the work on Taylor-type monetary policy rules is the importance that has been assigned in this literature to understanding how the evaluation of alternative policy rules involves assessing tradeoffs between various objectives, such as minimizing inflation, interest rates, and output volatility.

The analysis in this paper adds to this previous work on policy analysis by developing a theory of design limits to optimal policy. Specifically, we model the effects of alternative control rules on a state variable in the frequency domain. A frequency domain approach allows one to identify the frequency-specific components of the overall variance of the state variable. ${ }^{1}$ Our analysis then identifies the ways in which different control rules affect the contributions of fluctuations at each frequency to the overall variance. This approach allows us to develop an explicit characterization of the tradeoffs that exist between diminishing the variance contribution of one frequency and another. Examples of these tradeoffs in the control theory literature are the Bode and Poisson integral constraints; despite their importance in understanding the development of

\footnotetext{
${ }^{1}$ Such an interpretation is standard in time series analysis and derives immediately from the spectral representation of a time series.
} 
optimal policy, these types of constraints do not appear to have been previously exploited in the economics literature. The sorts of abstract arguments we develop have natural implications for contexts such as monetary policy design. Monetary policy rules are typically evaluated in terms of their ability to minimize fluctuations in state variables such as output and inflation.

In this context, the design limits we develop imply that policy rules that are efficacious in reducing low (high) frequency fluctuations in output and inflation will inevitably exacerbate the magnitude of high (low) frequency fluctuations, in a way that we make precise. These tradeoffs do not, of course, by themselves imply any particular ordering concerning the relative desirability of different policy rules. Rather, these tradeoffs imply that any ordering must carefully account for how the policymaker assesses the frequency-specific components of fluctuations for the outcomes of interest.

The design limits we describe are model-specific in the sense that a particular model produces a particular characterization of the frequency-specific tradeoffs that exist in stabilizing the state variable the policymaker wishes to control. In other words, different models imply different constraints on what a policymaker can achieve. As such, these limits are an important complement to work on policy design in the presence of model uncertainty ${ }^{2}$. Concerns over model uncertainty have motivated much recent research on the theoretical foundations of policy analysis; one example is the research program on robust policy construction initiated by Hansen and Sargent $(2001,2002,2003)$. This work focuses on local model uncertainty, i.e. model uncertainty that is local to a given baseline model. The interactions of policy design and model uncertainty in cases where model uncertainty is "global" in the sense that the space of potential models contains very different elements is explored in Brock, Durlauf, and West (2003). That paper uses an explicit decision-theoretic formulation which treats the true model as an unknown variable and computes expected policy effects by "integrating out" the variable. An important next step in studying policy design is the integration of the limits we describe here into environments with global model uncertainty; some initial discussion along these lines is found in Brock and Durlauf (2003).

\footnotetext{
${ }^{2}$ See Brock, Durlauf, and West (2003) and Onatski and Williams (2002) for conceptual and operational issues that arise in specifying model uncertainty in economic models.
} 
Our goal in this paper is to focus on basic ideas rather than formalism. Much of the work described here represents research in progress and we have felt free to be relatively speculative. Our hope is that the ideas we outline will stimulate others to pursue what we believe is an extremely promising research program.

\section{Design limits in linear systems: basic ideas}

This section considers the construction of optimal policies for systems with a scalar state variable $x_{t}$ and a scalar control $u_{t}$; such systems are known as Single Input Single Output or SISO systems. In our analysis, we assume that a policymaker wishes to minimize the unconditional variance of the state,

$$
E x_{t}^{2}
$$

The law of motion for the state is

$$
x_{t}=A(L) x_{t-1}+b u_{t-1}+\xi_{t}
$$

where $\xi_{t}$ represents an unobserved zero mean random variable with associated Wold moving average representation

$$
\xi_{t}=W(L) v_{t}
$$

The innovations $v_{t}$ are assumed to have a common variance $E v_{t}^{2}$; it is not necessary for our analysis that the error variances are constant. We focus on feedback rules for the control, i.e. rules of the form

$$
u_{t-1}=-F(L) x_{t-1}
$$


Given rules of the form (4), the law of motion (1) may be rewritten so that

$$
\begin{gathered}
x_{t}=A(L) x_{t-1}-b F(L) x_{t-1}+W(L) v_{t}= \\
\frac{W(L)}{1-L A(L)+b L F(L)} v_{t}
\end{gathered}
$$

if $1-L A(L)+b L F(L)$ is invertible, which we will assume.

In evaluating the effects of the control $u_{t}$ on the state $x_{t}$, it is useful to contrast (1) with the law of motion of the state variable when there is no control, i.e. the control is set equal to zero every period; we designate this process as $x_{t}^{N C}$. Eq. (2) of course implies that the state variable with no control obeys

$$
x_{t}^{N C}=A(L) x_{t-1}^{N C}+\xi_{t}
$$

Equation (6) is known in the control literature as the free dynamics of the system. In light of the loss function (1), one may interpret the optimal policy problem as identifying that choice of feedback rule that maximizes the difference between the variance of $x_{t}^{N C}$ and the variance of $x_{t}$.

In order to analyze the system (1)-(4), we will work in the frequency domain. To do this, for any lag polynomial $C(L)$ we denote the Fourier transform of its coefficients as $C(\omega)=\sum_{j=-\infty}^{\infty} c_{j} e^{-i j \omega}$. Using the notation $|C(\omega)|^{2}=C(\omega) C(-\omega)$, this allows us to express the variance of the state as

$$
E x_{t}^{2}=\frac{1}{2 \pi} \int_{-\pi}^{\pi} \frac{|W(\omega)|^{2} \sigma_{v}^{2}}{\left|e^{i \omega}-A(\omega)+b F(\omega)\right|^{2}} d \omega=\int_{-\pi}^{\pi} \frac{f_{\xi}(\omega)}{\left|e^{i \omega}-A(\omega)+b F(\omega)\right|^{2}} d \omega
$$


where $f_{\xi}(\omega)$ is the spectral density of $\xi_{t}{ }^{3}$

Eq. (7) provides a way of identifying how the control rule $-F(L)$ affects each of the frequency specific components of $E x_{t}^{2}$. To see this, multiply and divide the denominator of the integrand in (7) by $\left|e^{i \omega}-A(\omega)\right|^{2}$, so that

$$
\begin{gathered}
E x_{t}^{2}=\int_{-\pi}^{\pi} \frac{f_{\xi}(\omega)}{\left|e^{i \omega}-A(\omega)\right|^{2}\left|e^{i \omega}+b\left(e^{i \omega}-A(\omega)\right)^{-1} F(\omega)\right|^{2}} d \omega= \\
\int_{-\pi}^{\pi} \frac{f_{x^{N C}}(\omega)}{\left|e^{i \omega}+b\left(e^{i \omega}-A(\omega)\right)^{-1} F(\omega)\right|^{2}} d \omega
\end{gathered}
$$

where $f_{x^{N C}}(\omega)=\frac{f_{\xi}(\omega)}{\left|e^{i \omega}-A(\omega)\right|^{2}}$ by (6). Defining the function

$$
S(\omega)=\frac{1}{e^{i \omega}+b\left(e^{i \omega}-A(\omega)\right)^{-1} F(\omega)}
$$

(8) may be rewritten as

$$
E x_{t}^{2}=\int_{-\pi}^{\pi}|S(\omega)|^{2} f_{x^{N C}}(\omega) d \omega
$$

a representation that will prove to be of great use in understanding stabilization policy from a frequency domain perspective.

In control theory, $S(\omega)$ is known as the sensitivity function (cf. Kwakernaak and Sivan (1972), pg. 487). Equation (10) illustrates how the sensitivity function $S(\omega)$ plays a critical role in understanding policy design. The effects of a policy on a state are

\footnotetext{
${ }^{3}$ The sorts of calculations we make here are standard in the control theory literature; see Kwakernaak and Sivan (1972, chapter 6) for an excellent exposition.
} 
summarized by the sensitivity function; since $f_{x^{N C}}(\omega)$ does not depend on the policy rule, all effects of the policy rule are determined by the effect of $F(L)$ (and hence $F(\omega)$ ) on $S(\omega)$. This means that any restrictions that exist on the ability of the choice of $F(L)$ to determine the shape of $S(\omega)$ represent restrictions on how a policy can affect the variance of the state.

Eq. (10) indicates how different choices of the policy rule alter the way in which each frequency component of $f_{x^{N C}}(\omega)$ is transformed into an element of $f_{x}(\omega)$ and hence how each frequency-specific component of $x_{t}^{N C}$ translates into a frequency-specific component of $x_{t}$. Since $E\left(x_{t}^{N C}\right)^{2}=\int_{-\pi}^{\pi} f_{x^{N C}}(\omega) d \omega$ and $E x_{t}^{2}=\int_{-\pi}^{\pi} f_{x}(\omega) d \omega$, this transformation illustrates how the sensitivity function alters the frequency-specific variance contributions when $x_{t}^{N C}$ is transformed into $x_{t}$ by the feedback rule. When the policy rule has the implication that $|S(\omega)|^{2}<1$, then the contribution of fluctuations associated with frequency $\omega$ to $f_{x}(\omega)$ are smaller than their effect on the state variable would be if the control were set equal to zero, i.e. $f_{x^{N C}}(\omega)$. Hence, one way to think about policy design is that good policies will reduce the contributions of different values that are present in $f_{x^{N C}}(\omega)$ as the policy rule transforms this spectral density into $f_{x}(\omega)$ in a way to minimize the contributions of those frequencies where $f_{x^{N C}}(\omega)$ is relatively large.

What sorts of constraints exist on the choice of $S(\omega)$ which may be achieved by the choice of $F(L)$ ? It is intuitive that some such limits must exist, or else the policymaker would simply set $S(\omega)=0 \forall \omega \in[-\pi, \pi]$ and produce $E x_{t}^{2}=0$. (Such a rule is of course not realizable since the policymaker cannot condition on $v_{t}$ ) A full characterization of the limits that exist in generating $S(\omega)$ will provide a way of characterizing the fundamental limits that exist to feedback policies in this model. 
Within the control theory literature, there is an important result, known as the Bode integral constraint, which characterizes the limits which the state equation imposes on the mapping from $F(\omega)$ to $S(\omega)$. A discrete time version of this constraint is due to Wu and Jonckheere, (1992). In order to state this constraint, it is necessary to introduce a function $L(z)$ defined by

$$
L(z)=b F\left(z^{-1}\right)\left(z-A\left(z^{-1}\right)\right)^{-1}
$$

where for any lag polynomial $C(L)$ its associated $z$-transform $C(z)$ is defined by $C(z)=\sum_{j=-\infty}^{\infty} c_{j} z^{j} \cdot{ }^{4}$ We consider the case where $F(L)$ and $A(L)$ are polynomials of finite degree. By the fundamental theorem of algebra, this function may be written as a ratio of polynomials and factored into simple polynomials, therefore

$$
L(z)=c \frac{\prod_{i=1}^{m}\left(z-z_{i}\right)}{\prod_{i=1}^{n}\left(z-p_{i}\right)}
$$

where the constant $c$ is determined by the requirement that the system defined by (1) to (4) is stable. In the factorization, the quantities $z_{i}$ are the zeroes and the quantities $p_{i}$ are the poles of the function. The difference between the number of poles and the number of zeroes, $v=n-m$, is known as the relative degree of $L(z)$. We assume that $v \geq 1$. This background allows us to state the following theorem

\section{Theorem. Discrete Time Bode Constraint}

\footnotetext{
${ }^{4}$ We have shifted from Fourier to $z$-transforms in order to allow us to define the function $L(\cdot)$ on the entire complex plane, which is needed in the derivations of some of the results we employ.
} 
Assume that the controlled system is globally asymptotically stable and that $v \geq 1$. Then, there exists a non negative constant $K_{\text {Bode }}$ such that

$$
\int_{-\pi}^{\pi} \ln \left(|S(\omega)|^{2}\right) d \omega=K_{\text {Bode }}=4 \pi \sum_{i} \ln \left(\left|p_{u_{i}}\right|\right)
$$

where $p_{u_{i}}$ denotes an unstable pole of $L(z)$.

Pf. Wu and Jonckheere (1992).

The key features of this theorem are twofold. First, since $K_{\text {Bode }} \geq 0$ (which immediately follows from $\ln \left(\left|p_{u_{i}}\right|\right) \geq 0$ and the second equality in (13)), it is impossible for $|S(\omega)|<1 \forall \omega \in[-\pi, \pi]$; otherwise, $\int_{-\pi}^{\pi} \ln \left(|S(\omega)|^{2}\right) d \omega$ would be negative. From the perspective of eq. (7) this implies that it is impossible to design a policy such that $f_{x}(\omega) \leq f_{x^{N C}}(\omega) \forall \omega \in[-\pi, \pi]$ with strict inequality at some positive measure of frequencies. ${ }^{5}$ In order to achieve $f_{x}(\omega)<f_{x^{N C}}(\omega)$ for every element of one set of frequencies, it is necessary that $f_{x}(\omega)>f_{x^{N C}}(\omega)$ for elements of some other nontrivial set. Since $f_{x^{N C}}(\omega)$ represents the frequency-specific variance contributions when the control is identically equal to zero, this means that any nontrivial policy must increase the variance contributions at certain frequencies relative to the contributions in absence of the policy. Hence, there are fundamental tradeoffs between the variance contributions that collectively determine $E x_{t}^{2}$. An optimal policy may incur higher variance contributions at some frequencies in order to reduce variance contributions at others, but such tradeoffs are inevitable.

\footnotetext{
${ }^{5}$ Hence, the fact that it is impossible to choose a control such that $S(\omega)=0 \forall \omega \in[-\pi, \pi]$ is a special case of a much stronger set of restrictions on what feedback policies can achieve.
} 
When will $K_{\text {Bode }}>0$ ? A positive value for the constraint occurs when the polynomial $1-L A(L)$ has roots strictly outside the unit circle. Notice that unit root processes are compatible with $K_{\text {Bode }}=0$. We will treat $K_{\text {Bode }}=0$ as a leading case since many macroeconomic models are associated with $1-L A(L)$ polynomials whose roots are outside or on the unit circle.

The Bode integral constraint, in turn, provides a way of characterizing the optimal policy problem in the frequency domain. Let $p(\omega)=|S(\omega)|^{2}$. The optimal policy rule implicitly chooses the $p(\omega)$ that minimizes $E x_{t}^{2}$ subject to the constraint (13). Hence the optimal policy is determined by the

$$
\begin{gathered}
\min _{p(\omega)} \int_{-\pi}^{\pi} p(\omega) f_{x^{N C}}(\omega) d \omega+\lambda\left(K_{\text {Bode }}-\int_{-\pi}^{\pi} \ln p(\omega) d \omega\right)= \\
\lambda K_{\text {Bode }}+\int_{-\pi}^{\pi}\left(p(\omega) f_{x^{N C}}(\omega)-\lambda \ln p(\omega)\right) d \omega
\end{gathered}
$$

The first order necessary condition for this problem implies that for each frequency $\omega$ the optimal $p^{*}(\omega)$ fulfills

$$
f_{x^{N C}}(\omega)=\frac{\lambda}{p^{*}(\omega)} \rightarrow f_{x^{N C}}(\omega) p^{*}(\omega)=\lambda
$$

Eq. (15), in turn implies that the Fourier transform of the optimal feedback rule $-F^{*}(L),-F^{*}(\omega)$, is implicitly defined by

$$
p^{*}(\omega) f_{x^{N C}}(\omega)=\frac{|W(\omega)|^{2} \sigma_{v}^{2}}{\left|e^{i \omega}-A(\omega)+b F^{*}(\omega)\right|^{2}}=\lambda
$$

which in turn implies that the optimal feedback rule is 


$$
-F^{*}(L)=b^{-1}\left(L^{-1}-W(L) L^{-1}-A(L)\right)
$$

The optimal policy rule has a simple interpretation. Eq. (13) states the optimal policy rule shapes the function $p^{*}(\omega)$ so that $f_{x^{N C}}(\omega) p^{*}(\omega)$ is constant across frequencies. Recalling that $p(\omega)=|S(\omega)|^{2}$ and that $f_{x}(\omega)=|S(\omega)|^{2} f_{x^{x C}}(\omega)$, this means that the optimal policy rule converts the initial process $x_{t}^{N C}$ into a white noise process. In other words, any predictability in the state is eliminated under an optimal policy. This is precisely what one would expect from feedback rules of the form (3); predictability in the state is completely offset by the control.

Finally, we would note an intriguing implication of the Bode integral constraint for the analysis of policies in the presence of model uncertainty. The value of the constant $K_{\text {Bode }}$ is model-specific, as indicated by eq. (12). Models with unstable poles will thus produce different constraints on policy design than those where such poles are not present. This suggests a new avenue across which model uncertainty can affect optimal policy design as a policymaker will need to pay particular attention to the implications of a policy for those models where $K_{\text {Bode }}>0$ as such models imply that the cost of reducing variance at certain frequencies will be particularly high in the sense of creating especially large increases in variance at other frequencies.

\section{Applications}

In this section we apply the basic ideas associated with optimal policy design in the presence of the Bode integral constraint to illustrate some of the insights that may be produced by this framework.

\section{i. tradeoffs between low and high frequency fluctuations}


Suppose that a policymaker wishes to design a policy that works well when $f_{x^{N C}}(\omega)$ has the typical "Granger" spectral shape (Granger (1966), Sargent (1987)) so that $f_{x^{N C}}(\omega)$ is decreasing in $|\omega|$ with the possible exception of some local maxima and associated local increases around business cycle and seasonal frequencies. This spectral density shape suggests that a policymaker might want to design a feedback rule that is designed to reduce the variance contributions of frequencies in some interval $[-\bar{\omega}, \bar{\omega}]$ that makes a relatively large contribution to the overall value of $E x_{t}^{2}$. A strategy for doing this is to choose a feedback rule such that

$$
|S(\omega)| \leq M<1 \forall \omega \in[-\bar{\omega}, \bar{\omega}]
$$

Following eq. (10), such a policy means that the variance contribution of the frequencies in the interval $[-\bar{\omega}, \bar{\omega}]$ to $E x_{t}^{2}$ is

$$
\int_{|\omega| \leq \bar{\omega}}|S(\omega)|^{2} f_{x^{N C}}(\omega) d \omega \leq M^{2} \int_{|\omega| \leq \bar{\omega}} f_{x^{N C}}(\omega) d \omega
$$

How will such a policy perform in light of the Bode integral constraint? We focus on the case $K_{B o d e}=0$. The sort of strategy described by (18) provides good control with respect to those frequencies that contribute the most power to $E x_{t}^{2}$ under the assumption of the typical Granger spectral shape. However, this property comes at a price in the sense that it trades off good performance at low frequencies for poor performance at high frequencies.

The magnitude of the potential cost may be given a lower bound. If $K_{\text {Bode }}=0$, then by the Bode integral formula,

$$
0=\frac{1}{2} K_{\text {Bode }}=\int_{-\pi}^{\pi} \ln (S(\omega)) d \omega \leq 2 \bar{\omega} \ln (M)+\int_{\omega \notin[-\bar{\omega}, \bar{\omega}]} \ln (S(\omega)) d \omega
$$


which implies

$$
\sup _{\omega \notin[-\bar{\omega}, \bar{\omega}]}(|S(\omega)|) \geq M^{-\frac{\bar{\omega}}{\pi-\bar{\omega}}}>1
$$

Equation (21) illustrates the critical implication of the Bode integral constraint that $|S(\omega)|>1$ is unavoidable for some frequencies and thus shows how the success of a policymaker in controlling variance due to high spectral power at one frequency band requires exacerbation of the variance contribution of another frequency band. As a result, if we consider the variance contribution of frequencies outside the interval $[-\bar{\omega}, \bar{\omega}]$ to $E x_{t}^{2}$

$$
\int_{\omega \star[-\bar{\omega}, \bar{\omega}]}|S(\omega)|^{2} f_{x^{N C}}(\omega) d \omega
$$

it is clear that this contribution can be quite large, especially if $f_{x^{N C}}(\omega)$ has a local maximum in the set $|\omega|>\bar{\omega}$ as might occur, for example, via the Christmas cycle stressed by Barsky and Miron (1989). The implication of our analysis is that while policies of the form (18) would seem to be a natural implication of the assumption of the Granger spectral shape, such policies can prove to be ineffective (and even, at least in principle, counterproductive) if the implied costs of the policy in terms of increasing the variance contributions of frequencies $\omega \notin[-\bar{\omega}, \bar{\omega}]$ are such as to magnify local maxima in the spectral density of $x_{t}^{N C}$

\section{ii. local robustness analysis}

Within macroeconomics, one important new area of policy analysis has focused on the design of robust policies. Following the seminal contributions of Hansen and Sargent $(2001,2002,2003)$, this research addresses the question of how a policymaker can 
design policies that account for uncertainty about the model of the economy. Important recent contributions include Giannoni (2002), Massamiliano and Salmon (2002), Onatski and Stock (2002) and Tetlow and von zur Muehlen (2001).

Brock and Durlauf (2003) provide an analysis of local robustness for the system we have described in Section 2. We summarize their basic argument. Suppose that there exists model uncertainty with respect to the temporal structure of the innovations, $\xi_{t}$. We do this by introducing uncertainty about the spectral density $f_{\xi}(\omega)$. Specifically, relative to a baseline $\bar{f}_{\xi}(\omega)$, the true spectral density lies in the set defined by

$$
\int_{-\pi}^{\pi}\left(f_{\xi}(\omega)-\bar{f}_{\xi}(\omega)\right)^{2} d \omega \leq \varepsilon^{2}
$$

Let $f_{\xi}^{*}(\omega)$ denote (for the policymaker) the least favorable model in this set.

When $\varepsilon$ is small, arguments in Brock and Durlauf (2003) show that the least favorable model can be determined by identifying the marginal effect of an increase in $\bar{f}_{\xi}(\omega)$ on (23) and allocating the changes in a such a way that the ratio of the changes is equal to the ratio of the marginal effects on $E x_{t}^{2} .{ }^{6}$ The marginal effect of a change in the value of $\bar{f}_{\xi}(\omega)$ at a given frequency on $E x_{t}^{2}$, is $p^{*}(\omega)\left|e^{i \omega}-A(\omega)\right|^{2}$; this follows immediately from the frequency definition of the variance and the envelope theorem. Combining this with eq. (16), which describes the policymaker's feedback rule at the baseline spectral density, Brock and Durlauf (2003) show that the least favorable spectral density must fulfill

$$
f_{\xi}^{*}(\omega)=\bar{f}_{\xi}(\omega)+\varepsilon \frac{\bar{f}_{\xi}(\omega)^{-1}}{\left\|\bar{f}_{\xi}(\omega)^{-1}\right\|}+o(\varepsilon)
$$

${ }^{6}$ This is an indirect way of saying the ratio of the costs in changing $f_{\xi}(\omega)$ as embodied in (24) must equal the ratio of the benefits of the changes, and so is hardly a surprise from the perspective of basic price theory! 
Eq. (24) means that the least favorable model with respect to the baseline has the property that the difference between the baseline and the least favorable model is smaller when the baseline spectral density is relatively larger; conversely, the differences between the baseline and the least favorable model are larger when the baseline spectral density is relatively small. The intuition from this finding is driven by the logic of the optimal policy problem as affected by the Bode constraint. Those frequencies against which the optimal policy provides the smallest downweighting are those where the marginal harm of additional power is greatest. The example illustrates how the use of the Bode integral can facilitate calculations of robust versions of optimal policies.

\section{iii. policy design with uncertain policy effects}

While robustness analysis focuses on local model uncertainty, in the sense that the space of possible models represents a shroud around an initial baseline model, many forms of model uncertainty are clearly not local. One example of this occurs in the context of policy analysis when the effects of the control are uncertain. This type of problem is represented by Milton Friedman's (Friedman (1948)) famous criticism of activist monetary rule on the grounds that the effects of monetary policy are subject to "long and variable lags." We can formalize Friedman's idea in the context of a state equation of the form

$$
x_{t}=A(L) x_{t-1}+B(L) u_{t-1}+\xi_{t}
$$

and analyzing control design when $A(L)$ and $f_{\xi}(\omega)$ are known but $B(L)$ is unknown. Following our earlier strategy, we will exploit the fact that $f_{x^{N C}}(\omega)$ does not depend on $B(L)$. Let $\Theta$ denote the set of potential values of $B(L)$ with respect to which the policymakers seeks to design a "good" control. 
Suppose that $f_{x^{N C}}(\omega)$ displays the typical Granger spectral shape. Define a set of frequencies $[-\bar{\omega}, \bar{\omega}]$ such that $f_{\xi}(\omega) \leq e$ for $\omega \notin[-\bar{\omega}, \bar{\omega}]$ where $e$ is small positive number. Suppose that a policymaker designs a control so that the associated sensitivity function $S(\omega)$ has the property

$$
|S(\omega)|<1 \forall \omega \in[-\bar{\omega}, \bar{\omega}] \text { and } \forall B(L) \in \Theta
$$

Such a control implies

$$
\begin{gathered}
E x_{t}^{2}=\int_{-\pi}^{\pi}|S(\omega)|^{2} f_{x^{N C}}(\omega) d \omega \leq \\
\int_{-\bar{\omega}}^{\bar{\omega}} M f_{x^{N C}}(\omega) d \omega+\frac{1}{2 \pi} \int_{\omega \leftleftarrows-\bar{\omega}, \bar{\omega}]} e|S(\omega)|^{2} d \omega
\end{gathered}
$$

From the perspective of design limits, the question is how a policy which fulfills (26) performs relative to the last two terms in (27). Under the assumption that the system is stable for all lag structures in $\Theta$ (so that $K_{\text {Bode }}=0$ ) and letting $\left|S_{\infty}\right|=\sup _{\omega \in[-\pi, \pi]}|S(\omega)|$, the Bode integral constraint implies that

$$
\begin{gathered}
0=\int_{-\pi}^{\pi} \ln (S(\omega)) d \omega \leq \\
\int_{-\bar{\omega}}^{\bar{\omega}} \ln M d \omega+\int_{\omega \notin[-\bar{\omega}, \bar{\omega}]} \ln \left(\left|S_{\infty}\right|\right) d \omega=2 \bar{\omega} \ln M+(2 \pi-2 \bar{\omega}) \ln \left(\left|S_{\infty}\right|\right)
\end{gathered}
$$

which means

$$
\left|S_{\infty}\right| \geq M^{-\frac{\bar{\sigma}}{\pi-\bar{\omega}}}
$$

This formula illustrates how the strategy described by (26) can expose the policymaker to a large sensitivity peak; the inequality in (29) represents a lower bound on the weight assigned to some frequencies given the control rule. In parallel to our earlier arguments, from the perspective of designing good controls, the issue in minimizing 
frequencies in the way described by (26) is the effect of the control on $\int_{\omega \notin[-\bar{\omega}, \bar{\omega}]}|S(\omega)|^{2} f_{x^{N C}}(\omega) d \omega$. Eq. (29) places a lower bound on this term, i.e.

$$
\int_{\omega \notin[-\bar{\omega}, \bar{\omega}]}|S(\omega)|^{2} f_{x^{N C}}(\omega) d \omega \geq M^{-\frac{\bar{\omega}}{\pi-\bar{\omega}}} \int_{\omega \notin[-\bar{\omega}, \bar{\omega}]} f_{x^{N C}}(\omega) d \omega
$$

so, policy rules that account for the uncertainty in the effects of the control on the state in minimizing low frequency fluctuations can potentially involve serious problems at higher frequencies.

\section{iv. measurement error}

The theory of design limits may also be applied to study how to design good controls in the presence of measurement error. Problems of this type naturally arise in monetary policy contexts, for example, where measurement of relevant variables such as potential output or the natural rate of unemployment can be problematic; see Orphanides and Williams (2002) for a recent example.

To see how design limits apply to measurement error, suppose that the true state variable $x_{t}$ obeys the process described by eq. (2) but that state is unobservable; the measured level of the state, $x_{t}^{m}$ is

$$
x_{t}^{m}=x_{t}+n_{t}
$$

where $n_{t}$ is measurement error. Control equations can of course only be constructed as functions of observables so the control takes the form

$$
u_{t}=-F(L) x_{t}^{m}
$$


To understand how measurement error affects the choice of control rule, we assume that $\xi_{t}$ is zero. ${ }^{7}$ In this case, the variance of the state is

$$
E x_{t}^{2}=\int_{-\pi}^{\pi}|S(\omega)-1|^{2} f_{n}(\omega) d \omega
$$

which indicates that the control should be chosen so that $|S(\omega)-1|$ is relatively small when $f_{n}(\omega)$ is relatively large. Notice that relative to the earlier formulas one is now concerned with the spectral density of the measurement error and not the true state. Also, the weighting scheme is no longer $|S(\omega)|^{2}$ but $|S(\omega)-1|^{2}$. The difference in these weights reflects the fact that the policy rule wishes to damp out the effects of the measurement error in inducing volatility in $x_{t}$ because of the feedback rule, not because of volatility that is intrinsic to $x_{t}^{N C}$.

\section{v. non-time separable preferences}

The frequency domain perspective on policy limits provides some interesting insights into the design of optimal policies in the presence of non-time separable preferences (NTS). As argued in recent work such as Otrok (2001), Otrok, Ravikumar, and Whiteman (2002), the standard time separable preferences used in macroeconomics may fail to properly account for the fact that economic actors are sensitive to the frequency of shocks. Accounting for this may have important macroeconomic implications. For example, Otrok, Ravikumar, and Whiteman (2002) show how non-time separability can explain the equity premium puzzle. We apply the analysis of Section 2 to the design of policies in the presence of NTS preferences. We do this by contrasting a standard set of time separable preferences

\footnotetext{
${ }^{7}$ One might object that if $f_{\xi}(\omega)=0 \forall \omega \in[-\pi, \pi]$, there is no control problem that needs to be addressed. Our purpose in this assumption is to highlight an implication of measurement error, not to provide a realistic stabilization problem.
} 


$$
(1-\beta) \sum_{j=0}^{\infty} \beta^{j} x_{t+j}^{2}
$$

with non-time separable preferences of the form

$$
(1-\beta) \sum_{j=0}^{\infty} \beta^{j}\left(x_{t+j}-\delta x_{t+j-1}\right)^{2}
$$

These alternative preference structures illustrate that the difference between the payoffs under time separable and non-separable cases is determined by the difference between $E x_{t}^{2}$ and $E\left(x_{t}-\delta x_{t-1}\right)^{2}$. From eq. (7), it is immediately the case that $E\left(x_{t}-\delta x_{t-1}\right)^{2}=E\left((1-\delta L) x_{t}\right)^{2}$ may be expressed as

$$
\begin{gathered}
E\left(x_{t}-\delta x_{t-1}\right)^{2}= \\
\int_{-\pi}^{\pi}\left|e^{i \omega}-\delta\right|^{2}|S(\omega)|^{2} f_{x^{N C}}(\omega) d \omega= \\
\int_{-\pi}^{\pi} \frac{\left|e^{i \omega}-\delta\right|^{2}}{\left|e^{i \omega}-(A(\omega)-b F(\omega))\right|^{2}} f_{\xi}(\omega) d \omega
\end{gathered}
$$

where we have used the identities $\left|1-\delta e^{-i \omega}\right|^{2}=\left|e^{-i \omega}\left(e^{i \omega}-\delta\right)\right|^{2}$ and $\left|e^{-i \omega}\right|^{2}=1$. It is straightforward to verify, using the analysis underlying (16) and (17) that $F(L)=b^{-1} A(L)$ when $\delta=0$.

To see how non-time separable preferences affect optimal policy, we consider the special case where $A(L)=a_{0}$ and $f_{\xi}(\omega)=\frac{1}{2 \pi}$; we also normalize so that $b=1$. For this case, the optimal feedback rule takes the form

$$
u_{t-1}=-F^{*}(\delta) x_{t-1}
$$


where the coefficient $F(\delta)$ reflects the dependence of the feedback parameter on $\delta$. To understand this dependence, (25) implies that for each choice of the feedback parameter F

$$
\begin{gathered}
E\left(x_{t}-\delta x_{t-1}\right)^{2}=\int_{-\pi}^{\pi} \frac{\left|e^{i \omega}-\delta\right|^{2}}{\left|e^{i \omega}-\left(a_{0}-F\right)\right|^{2}} d \omega= \\
\int_{-\pi}^{\pi} \frac{1-2 \delta \cos \omega+\delta^{2}}{1-2\left(a_{0}-F\right) \cos \omega+\left(a_{0}-F\right)^{2}} d \omega
\end{gathered}
$$

Hence, the effects of habit persistence on optimal policy are determined by the behavior of $1-2 \delta \cos \omega+\delta^{2}$. This function is monotonically decreasing in $|\omega|$ if $\delta>0$.

In order to solve for $F(\delta)$, we proceed as follows. The second integral in (38) may, using a formula in Gradshteyn and Ryzhik (2000, formula 2.554(2), pg. 169) be rewritten as

$$
\begin{gathered}
\int_{-\pi}^{\pi} \frac{1-2 \delta \cos \omega+\delta^{2}}{1-2\left(a_{0}-F\right) \cos \omega+\left(a_{0}-F\right)^{2}} d \omega= \\
\frac{\pi\left(1+\delta^{2}-2 \delta\left(a_{0}-F\right)\right)}{1-\left(a_{0}-F\right)^{2}}
\end{gathered}
$$

Differentiation of (39) with respect to $\delta$ produces a quadratic equation. Setting this quadratic equal to 0 in order to minimize (39) and choosing the relevant root leads to the formula

$$
F(\delta)=a_{0}-\delta
$$

To understand the intuition behind this formula, consider a feedback rule $-F(L)$ such that 


$$
A(L)-b F(L)=\delta
$$

When $\xi_{t}$ is uncorrelated, this rule implies that all correlation in $x_{t}-\delta x_{t-1}$ has been eliminated by the feedback rule. Following the analysis of (17), this means the rule is optimal.

\section{Multivariate systems}

Our discussion thus far has focused on SISO models. There is also a rich literature on multiple input multiple output (MIMO) systems that is of particular importance in contexts where the policymaker wishes to control several state variables, as occurs for a monetary authority who cares about output and inflation volatility. The theory of design limit for MIMO systems is relatively less developed than for SISO systems; important contributions include Chen (1995) and Chen and Nett (1995). We consider the case where there are two states and one control.

We consider a system for two state variables $x_{1, t}$ and $x_{2, t}$. The equations for these states are

$$
x_{1, t}=a_{11} x_{1 . t-1}+a_{12} x_{2, t-1}+b_{1} u_{t-1}+\xi_{1, t}
$$

and

$$
x_{2, t}=a_{21} x_{1 . t-1}+a_{22} x_{2, t-1}+b_{2} u_{t-1}+\xi_{2, t}
$$

which in matrix form is

$$
x_{t}=A x_{t-1}+b u_{t-1}+\xi_{t}
$$

Models of this type are common in the macroeconomics literature. For example, Onatski and Stock (2001) consider a model of this type in which a policymaker attempts 
to stabilize inflation and the output gap using a short run interest rate as the control. Onatski and Stock restrict their analysis to evaluate policies that lie in a set of Taylor rules. In our context, this would mean that policies are taken from the set $T$ defined by

$$
T=\left\{u_{t} \text { such that } u_{t}=g_{1} x_{1, t}+g_{2} x_{2, t}=g^{\prime} x_{t}\right\}
$$

Our objective is to derive some comparisons with the SISO case we have already discussed. To do this, one first considers the matrix generalization of the sensitivity function. The $2 \times 2$ sensitivity matrix $S(\omega)$ is defined as

$$
S(\omega)=\left(I+\left(I+\left(e^{i \omega} I-A\right)^{-1}\right) b g^{\prime}\right)^{-1}
$$

where $I$ is the identity matrix.

We have been unable to uncover an explicit formula for the Bode integral constraint for multivariate discrete time systems that represents a direct generalization of (13), although there are a number of results available that consider design limits in such systems (cf. Chen and Nett $(1993,1995)$ and Chen (1995)). We conjecture that the multivariate analog of the sensitivity formula is

$$
K_{\text {Bode }}=\int_{-\pi}^{\pi} \ln \left(\operatorname{det}(S(\omega))^{2}\right) d \omega=4 \pi \sum_{i} \ln \left(\left|p_{u_{i}}\right|\right)
$$

In this case, the terms $p_{u_{i}}$ represent unstable roots of the matrix $A$ in eq. (44). Letting $S(z)$ denote the $z$-transform that corresponds to the sensitivity matrix defined by (46)

$$
\operatorname{det} S(z)=\frac{\Pi\left(z-p_{i}\right)}{\Pi\left(z-z_{i}\right)}
$$


While we are in the process of attempting to develop a proof of (47), we emphasize that we have not done so. ${ }^{8}$ At the same time, the arguments we make below can be readily modified if we turn out to be incorrect in this conjecture and so we proceed on the basis that (47) holds in order to illustrate the design limits ideas.

In order to see how (47) constrains the design of policy, we employ the relationship

$$
\sum_{k=1}^{2} \int_{-\pi}^{\pi} \ln |\operatorname{det}(S(\omega))|^{2} d \omega=\sum_{k=1}^{2} \int_{-\pi}^{\pi} \ln \left(\sigma_{k}(S(\omega))\right) d \omega
$$

where, for a fixed $\omega, \sigma_{k}(S(\omega))$ denotes the singular values of $S(\omega) .{ }^{9}$ Let the norm $|S|_{\infty}$ be defined as the largest singular value of $S(\omega)$ where $\omega$ is allowed to vary between $-\pi$ and $\pi$. In analogy to (18), one can consider the effects of policies that impose

$$
|S|_{\infty} \leq M \forall \omega \in[-\bar{\omega}, \bar{\omega}]
$$

This expression is more complicated than (18) because we are now working with a sensitivity matrix function. If one combines (47) and (50),

$$
0 \leq K_{\text {Bode }}=\sum_{k=1}^{2} \int_{-\pi}^{\pi} \ln \left(\sigma_{k}(S(\omega))\right) d \omega \leq 2 \int_{-\pi}^{\pi} \ln \left(\sup _{\omega \in[-\pi, \pi]} \sigma_{2}(S(\omega))\right)
$$

which implies

\footnotetext{
${ }^{8}$ The continuous time analog to (48) is readily available in the control literature, cf. Skogestad and Postlethwaite (1996, eq. 6.3, pg. 215).

${ }^{9}$ The singular value of a complex valued $2 \times 2$ matrix $S$ are the square roots of the eigenvalues of the matrix $S S^{H}$ where $S^{H}$ is the complex transpose of $S$, cf. Skogestad and Postlethwaite (1996, pg. 503). The singular values are ordered so that for each $\omega$, $\sigma_{1}(S(\omega)) \leq \sigma_{2}(S(\omega))$.
} 


$$
0 \leq 2 \bar{\omega} \ln M+(2 \pi-2 \bar{\omega}) \ln |S|_{\infty}
$$

since $|S|_{\infty}=\sup _{\omega \in[-\pi, \pi]} \sigma_{2}(S(\omega))$. Therefore, it is the case that

$$
|S|_{\infty} \geq M^{-\frac{\bar{\omega}}{\pi-\bar{\omega}}}
$$

This bound indicates how the basic principles we have described for univariate systems apply to multivariate systems, namely, reduction of variance associated with low frequencies can produce high variance contributions from high frequencies. In principle, it should be possible to obtain tighter bounds on the effect of strategy (50) on the magnification of high frequency components using methods such as those developed in Zhou, Doyle, and Glover (1998, chapter 6); we leave this to future work.

While the basic principles of design limits extend naturally from SISO to MIMO systems, there are interesting differences because design limits apply to frequencyspecific components of variance both within and across the state variables. Put differently, in the SISO case, design limits always expose a policymaker to undesirable effects outside the range of frequencies which are targeted by the policymaker. In the MIMO case these undesirable effects themselves will embody tradeoffs with respect to the different state variables. This issue is well exposited in Skogestad and Postlethwaite (1996, chapter 6). ${ }^{10}$

\footnotetext{
${ }^{10}$ There are also important technical differences between SISO and MIMO systems. First, in the SISO case, if the controlled system is stable, then $\ln (|S(\omega)|)$ is harmonic outside the unit disk, whereas in MIMO systems, even if $S(\omega)$ is analytic, $\ln (|S(\omega)|)$ is not harmonic in general where, for the case of matrix $S(\omega),|S(\omega)|$ denotes the largest singular value of $S(\omega)$ at $\omega$ (Hara and Sung (1989, pg. 890)). Second, both the locations of unstable poles and zeros as well as their directions affect the constraints in MIMO systems (Hara and Sung (1989, pg. 890)). Similar differences are discussed in Chen (1995) and Chen and Nett $(1993,1995)$.
} 


\section{Summary and conclusions}

This paper has attempted to outline some basic issues that arise in the design of optimal policies in dynamic economic systems. Borrowing ideas from the control engineering literature, it is possible to precisely delineate the limitations that a policymaker faces in designing feedback policies. These limits, which are summarized by a remarkable formula known as the Bode integral constraint, illustrate how any feedback policy is forced to make tradeoffs among the frequency-specific components of the state variables that a policymaker wishes to stabilize. We have suggested how these tools can elucidate policy design issues that arise in the presence of local and global model uncertainty as well as in the face of measurement error. These applications represent only a hint of the potential of these methods for understanding policy design in macroeconomics and other areas of economics. 


\section{Bibliography}

Barsky, R. and J. Miron, (1989), "The Seasonal Cycle and the Business Cycle," Journal of Political Economy, 97, 3, 503-534.

Brock, W. and S. Durlauf, (2003), "Local Robustness Analysis: Theory and Application," mimeo, Department of Economics, University of Wisconsin.

Brock, W., S. Durlauf, and K. West (2003), "Policy Evaluation in Uncertain Economic Environments (with discussion)," Brookings Papers on Economic Activity, 1, 235-322.

Chen, J., (1995), "Sensitivity Integral Relations and Design Trade-Offs in Linear Multivariate Feedback Systems," IEEE Transactions on Automatic Control, 40, 10, 17001716.

Chen, J. and C. Nett, (1993), "Bode Integrals for Multivariable Discrete Time Systems," Proceedings of the 32 Conference on Decision and Control, 811-816.

Chen, J. and C. Nett, (1995), "Sensitivity Integrals for Multivariate Discrete-Time Systems," Automatica, 31, 8, 1113-1124.

Friedman, M., (1948), "A Monetary and Fiscal Framework for Economic Stability," American Economic Review, 38, 245-264.

Friedman, M., (1951), "Comments on Monetary Policy," Review of Economics and Statistics, 33, 3, 186-191.

Giannoni, M., (2002), "Does Model Uncertainty Justify Caution? Robust Optimal Monetary Policy in a Forward-Looking Model," Macroeconomic Dynamics, 6, 111-144.

Granger, C., (1966), "The Typical Shape of an Economic Variable," Econometrica, 34, 1, 150-161.

Gradshteyn, I. and I. Ryzhik, (2000), Table of Integrals, Series, and Products, Sixth Edition, San Diego: Academic Press.

Hansen, L. and T. Sargent, (2001), "Acknowledging Misspecification in Macroeconomic Theory," Review of Economic Dynamics, 4, 519-35.

Hansen, L. and T. Sargent, (2002), Robust Control and Economic Model Uncertainty. Book manuscript, Hoover Institution, Stanford University and forthcoming, Princeton University Press.

Hansen, L. and T. Sargent, (2003) "Robust Control of Forward-Looking Models," Journal of Monetary Economics, 50, 581-604. 
Hara, S. and H. Sung, (1989), "Constraints on Sensitivity Characteristics in Linear Multivariate Discrete-Time Control Systems," Linear Algebra and its Applications, 122124, 889-919.

Kwakernaak, H. and R. Sivan, (1972), Linear Optimal Control Systems, New York: John Wiley and Sons.

Massamiliano, M. and M. Salmon, (2002), "Robust Decision Theory and the Lucas Critique," Macroeconomic Dynamics, 6, 1, 167-185.

Onatski, A. and J. Stock, (2002), "Robust Monetary Policy Under Model Uncertainty in a Small Model of the U.S. Economy," Macroeconomic Dynamics, 6, 85-110.

Onatski, A. and N. Williams, (2003), "Modeling Model Uncertainty," Journal of the European Economic Association, forthcoming.

Orphanides, A. and J. Williams, (2002), "Robust Monetary Policy Rules with Unknown Natural Rates," Brookings Papers on Economic Activity, 2, 63-118.

Otrok, C., (2001), "Spectral Welfare Cost Functions," International Economic Review, $42,2,345-367$.

Otrok, C., B. Ravikumar, and C. Whiteman, (2002), "Habit Formation: A Resolution of the Equity Premium Puzzle, Journal of Monetary Economics, 49, 1261-1288.

Sargent, T., (1987), Macroeconomic Theory, San Diego: Academic Press.

Sims, C., (2001), "Pitfalls of a Minimax Approach to Model Uncertainty," American Economic Review, 91, 51-4.

Skogestad, S. and I. Postlethwaite, (1996), Multivariable Feedback Control: Analysis and Design, New York: John Wiley.

Svensson, L., (1996), "Commentary: How Should Monetary Policy Respond to Shocks While Maintaining Long Run Price Stability?," in Achieving Price Stability, Federal Reserve Bank of Kansas City, 181-195.

Svensson, L., (2000), "Robust Control Made Simple," mimeo, Department of Economics, Princeton University.

Taylor, J., (1993), "Discretion Versus Policy Rules in Practice," Carnegie-Rochester Conference Series on Public Policy, 39, 195-214.

Taylor, J., (ed.), (1999), Monetary Policy Rules, Chicago: University of Chicago Press. 
Tetlow, R. and P. von zur Muehlen, (2001), "Robust Monetary Policy With Misspecified Models: Does Model Uncertainty Always Call for Attenuated Policy?," Journal of Economic Dynamics and Control, 25, 6-7, 911-949.

Wu, B.-F. and E. Jonckheere, (1992), "A Simplified Approach to Bode's Theorem for Continuous and Discrete Time Systems," IEEE Transactions on Automatic Control, 37, $100,1797-1802$.

Zhou, K., J. Doyle, and K. Glover, (1998), Robust and Optimal Control, Englewood Cliffs, New Jersey: Prentice Hall. 